Cinémas

Revue d'études cinématographiques

Journal of Film Studies

\title{
Le Documentaire, une fiction (pas) comme les autres
}

\section{Geneviève Jacquinot}

Volume 4, numéro 2, hiver 1994

Le Documentaire

URI : https://id.erudit.org/iderudit/1001023ar

DOI : https://doi.org/10.7202/1001023ar

Aller au sommaire du numéro

Éditeur(s)

Cinémas

ISSN

1181-6945 (imprimé)

1705-6500 (numérique)

Découvrir la revue

Citer cet article

Jacquinot, G. (1994). Le Documentaire, une fiction (pas) comme les autres.

Cinémas, 4(2), 61-81. https://doi.org/10.7202/1001023ar
Résumé de l'article

En vue de chercher à expliquer comment un film documentaire (n') est (pas) une fiction comme les autres, l'auteur analyse, après avoir réexaminé certaines oppositions connues (telles réel/imaginaire, histoire/récit, vrai/faux,

objectivité/subjectivité, etc.), trois exemples documentaires différents où la «fiction » est utilisée en vue d'augmenter ce que l'on appelle l'« effet de réel ». 


\section{Le Documentaire, une fiction (pas) comme les autres}

\section{Geneviève Jacquinot}

\section{RÉSUMÉ}

En vue de chercher à expliquer comment un film documentaire ( $n$ ') est (pas) une fiction comme les autres, l'auteur analyse, après avoir réexaminé certaines oppositions connues (telles réel/imaginaire, histoire/récit, vrai/faux, objectivité/subjectivité, etc.), trois exemples documentaires différents où la "fiction" est utilisée en vue d'augmenter ce que l'on appelle l'«effet de réel».

\section{ABSTRACT}

In order to try to explain how a documentary film is (not) a fiction like others, after having reexamined some usual oppositions (such as real/imaginary, story/narrative, true/false, objectivity/subjectivity, etc.), the author analyses three different documentary cases where "fiction" is used in order to increase what we call "the effect of real."

En empruntant ce titre à l'une des parties du dernier livre de Bill Nichols, Representing Reality, je m'inscris d'emblée, avec lui, sans tomber dans le "mythe de la transparence", contre une mode actuelle qui voudrait accréditer l'idée qu'après tout - et c'est dans ce "tout" que réside le problème -, il n'y a pas de différence entre documentaire et fiction.

Cette opposition, qui structure toute l'histoire du cinéma et qui a été, comme elle se devait, réinterrogée au nom de divers principes et réalités, se voit tout simplement retournée par la pensée dite postmoderne, qui en vient à affirmer que le cinéma, (et plus généralement l'audiovisuel) ne peut être que de fiction. 
S'il est vrai que rares seraient ceux qui, à l'heure actuelle, spécialistes ou non du cinéma, s'aventureraient encore dans des définitions aussi tranchées que "documentaire, film instructif, s'opposant à la fiction" ${ }^{1}$, il est vrai aussi que les grilles d'émissions télévisées continuent de distinguer ces deux catégories de programmes comme le refont ${ }^{2}$, plus récemment encore, les programmations cinématographiques du type «Documentaires sur grand écran». Vrai aussi que se multiplient les productions qui se situent "entre documentaire et fiction" comme l'indiquent bon nombre de critiques cinématographiques ou télévisuelles ${ }^{3}$. Tout cela témoigne assez de la persistance sinon de la pertinence de l'opposition et, encore plus, de la nécessité d'interroger ce que recouvre, tout autant que cette traditionnelle distinction, la nouvelle "non-distinction" qu'on tend à lui substituer. C'est ce que nous voudrions faire dans ce qui va suivre, en analysant trois exemples différents de "fictionnalisation" dans des documentaires ${ }^{4}$, après avoir pris soin, en un premier temps, d'examiner les termes dans lesquels se trouve posée cette distinction/nondistinction et les glissements de sens grâce auxquels elle peut se perpétuer.

\section{De la nécessité de dépasser l'opposition documentaire/fiction}

On peut montrer de diverses façons que le documentaire s'oppose à la fiction : dans la généalogie du cinéma ("la nature prise sur le vif" des frères Lumière opposée au "spectacle cinématographique» de Méliès); dans les diverses définitions qui en sont données (jusques et y compris ce terme générique qui définit négativement le documentaire comme "non-fiction"); dans l'institution cinématographique elle-même, avec ses circuits spécifiques de production et de diffusion et les réalités économiques correspondantes; dans la biographie des cinéastes, où le documentaire a souvent fait figure de banc d'essai pour futurs réalisateurs en mal de moyens humains et matériels, et, en tout état de cause, "en attendant mieux»; enfin dans la conscience individuelle et collective puisque aussi bien un spectateur qui entrerait dans une salle et à qui on projetterait un film sans lui dire si c'est un documentaire ou une fiction serait capable de le décider après quelques minutes - chacun a pu en faire l'expérience. 
Inversement, on peut tout aussi bien montrer, avec d'autres arguments, empruntés à d'autres pertinences bien sûr, que le documentaire ne s'oppose pas à la fiction, ne serait-ce, pour commencer, que parce que dans toute fiction, il peut y avoir un aspect documentaire ${ }^{5}$ et plus généralement parce qu'il y a du document dans la fiction "pour la bonne raison que la caméra (c'est plus fort qu'elle) enregistre ce qu'on met devant elle, tout ce qu'on met devant elle» (Daney, p. 166). D'ailleurs, pour le grand documentariste belge Henri Storck, le plus grand documentariste fut Hitchcock, parce qu'il a donné du décor de notre vie et des objets qui jouent un rôle important dans notre vie, des images absolument saisissantes; et pour le critique Serge Daney, c'était, au moins pour la France, Jacques Tati parce qu' «il lançait des coups de sonde d'ethnologue rigolard dans la société française (...) la voyant comme une tribu sympathique mais en perpétuel devenir» (p. 134).

Le documentaire ne s'oppose pas si facilement à la fiction aussi parce que le documentaire n'est pas toujours le meilleur document - on cite souvent, à ce propos, l'authentique témoignage des luttes de la résistance italienne du film Rome ville ouverte (1944-46) dont Rossellini put dire un jour en plaisantant, à moitié, que ce film «fit plus que tous les discours de notre ministre des Affaires étrangères pour que l'Italie retrouve sa place dans le concert des nations" (cité par Sadoul dans son dictionnaire des films); parce qu'enfin, comme l'a très bien montré Roger Odin, sous certaines déterminations externes, pragmatiques, un film de fiction peut donner lieu à une lecture "documentarisante" (et inversement) - j'ai pu le constater moi-même, notamment lors d'une enquête effectuée dans un quartier populaire d'Abidjan où, pour une partie non négligeable des spectateurs ivoiriens, le cinéma qu'ils voyaient (essentiellement des films indiens, de karaté, des westerns et des policiers) était considéré comme «un moyen d'information et de culture", sans parler du cinéma pornographique assimilé à "des films d'éducation sexuelle». N'est pas toujours éducatif le film qu'on pense, notamment dans les pays où l'école n'est pas le bien de tous!

Si l'on veut donc sortir de cette opposition figée entre documentaire et fiction aussi bien que de sa dénégation illusoire et à 
la mode, il faut déplacer la question et la porter ailleurs, dans une interrogation antérieure à la classification institutionnelle par genres, à savoir dans les relations que le cinéma entretient avec la réalité qui lui est extérieure et où, comme toute l'histoire du cinéma et déjà de la télévision le prouve, ces deux modalités de relation au réel se sont toujours combinées, selon des degrés d'interpénétration divers, à la manière des deux serpents d'un caducée, comme le disait si pertinemment Alain Resnais, qui l'a sans doute, plus que tout autre, prouvé dans toute son œuvre.

L'opposition documentaire/fiction doit être dépassée, non pour les raisons "morales" qu'évoquaient les premiers documentaristes $^{6}$, mais pour des raisons théoriques, car ce sont deux façons différentes d'interroger le monde et d'en rendre compte, et s'il ne faut cesser de rappeler qu'il n'y a pas de supériorité ontologique de l'une par rapport à l'autre, en revanche, il y a bien entre elles des différences qu'il ne sert à rien de nier.

\section{De quelques glissements progressifs de sens}

Comme l'a reconnu à juste titre François Jost, non seulement cette traditionnelle définition du documentaire a contrario de la fiction est critiquable d'un point de vue épistémologique, mais elle n'est même pas constructive puisque qu'elle consiste à opposer à un concept à définir (le documentaire) un concept flou ( la fiction) - j'ajouterai : tous deux étant pris entre divers couples d'opposition indistinctement et alternativement allégués, selon les besoins de la démonstration : discours/récit, réel/imaginaire, vrai/faux, objectivité/subjectivité, etc.

Précisons : un récit peut être "réel" (renvoyer à des objets, des personnages ou des événements réels) ou "fictif" (renvoyer à des objets, des personnages ou des événements nés de l'imagination), c'est donc l'identification du récit à la fiction qui fait problèmeil peut y avoir des récits du réel (factuels comme on dit), et donc le récit peut faire partie du documentaire. Inversement un documentaire peut renvoyer à des objets, des événements ou des personnages réels, reconstitués ${ }^{7}$ ou fictifs (imaginaires), c'est donc l'identification $d u$ documentaire au non-imaginaire qui fait problème. La distinction se situe au niveau du discours : ce qu'on pourrait dire de la façon suivante : ce qui différencie le documentaire de la fiction, ce n'est pas la nature des objets rapportés mais 
la nature de l'acte de s'y rapporter, fictif dans un cas, réel dans l'autre.

Le documentaire est opposé à la fiction qui est assimilée à la narration ou histoire racontée. Là, c'est la pertinence narratologique qui est en question et il n'est pas inutile de rappeler qu'un "récit" ${ }^{8}$ (narrative en anglais) construit une "histoire" (story) par le biais de la "narration" (narration en anglais) : autrement dit, que le récit est donc l'«énoncé» dans sa matérialité littéraire ou cinématographique (la diégèse) qui prend en charge l'histoire (même réduite dans le cas d'une action non mouvementée et non dramatique), par le jeu de l'énonciationnarration ${ }^{9}$ ou "acte narratif producteur", identifiable par les traces laissées dans le texte narratif, et ce n'est que par extension qu'on appelle narration la situation réelle ou fictive dans laquelle la narration prend place.

Le documentaire peut donc être narratif, et s'il l'est moins souvent et surtout moins totalement que la fiction - comme l'a bien montré William Guynn, c'est justement parce que son objectif prioritaire (pertinence pragmatique) n'est pas de raconter des histoires ${ }^{10}$, nous y reviendrons, même lorsqu'il insère ou utilise des récits d'événements réels ou imaginaires.

Le documentaire est souvent aussi opposé à la fiction dans la relation au vrai opposé au faux ou à l'objectivité opposée à la subjectivité. Or il n'y a rien dans le discours et donc dans le textuel (qu'il soit écrit ou filmique) qui porte la trace infaillible de "vérité" ou de "mensonge», car tous les signes peuvent être simulés, comme le prouve assez le célèbre pastiche des "premiers films" des vedettes du documentaire mondial, réalisé par PierreOscar Lévy, dans Premiers mètres, en $1984^{11}$. Quand on affirme que documentaire et fiction sont aussi mensongers l'un que l'autre, dans la mesure où il est impossible d'être objectif ou bien parce que l'image est toujours manipulation, dans un cas, on confond la subjectivité du discours et l'invention de la fiction; dans l'autre, on confond manipulation et falsification ou tromperie, car la mise en cadre ou la mise en scène est par définition décomposition et réorganisation de la matière vivante, sans parler du montage. C'est donc l'assimilation du documentaire à l'objectivité et à la vérité par opposition à la subjectivité et à la fausseté de la fiction qui pose problème, car la subjectivité renvoie 
ou à une caractéristique du discours (qui s'oppose bien alors à l'objectivité) etlou à une manifestation de l'invention (qui s'oppose alors à la réalité factuelle).

Enfin, s'il est vrai que "tout film est de fiction" comme l'a très bien montré Christian Metz dans la mesure où "ses matières de l'expression irréalise ce qu'il représente» (p. 63) (niveau de pertinence sémiologique) - le signifiant cinématographique est bien imaginaire - on ne peut en déduire si facilement qu'il n'y a pas de différence entre documentaire et fiction : le faire c'est, comme l'a dénonçé François Jost, "glisser des propriétés sémiotiques d'un langage aux objets discursifs qu'il permet d'engendrer (...) comme si, du seul fait que les mots ne sont pas les choses, on en concluait que tout discours verbal est fictif" (p. 40).

Même lorsque Gérard Leblanc renchérit en affirmant, à juste titre, que tout le cinéma est doublement fictionnel «non seulement parce que la réalité filmée est toujours déjà une représentation" (p. 45), mais parce que, quel que soit leur degré d'élaboration artistique, la plupart des films "partagent l'illusion d'épuiser la réalité par le visible» (p. 45) (d'où ce qu'il appelle les "fictions du visible" et les "fictions de la réalité»), il retrouve, à un deuxième niveau, cette distinction puisqu'il dit que cette réduction de la réalité au visible joue aussi bien pour la fiction que pour le documentaire.

On pourrait décliner ainsi bien d'autres fausses distinctions ou assimilations hâtives, et l'on s'aperçoit, ainsi, que la traditionnelle opposition entre documentaire et fiction tout autant que la nouvelle non-distinction ne fonctionnent, en réalité, qu'au prix d'innombrables glissements progressifs des sens... et du mélange de niveaux de pertinence. D'où la nécessité de se demander ce qui fait du documentaire une fiction (pas) comme les autres. Nous nous proposons de commencer à répondre à cette question $^{12}$ en examinant la fonction de la fiction dans le documentaire.

Des diverses modalités de fictionnalisation du documentaire

1. De La SÉQUeNCE FICTIONNELLE COMME SIMULATION SUBJECTIVE

Le premier documentaire, Saîd Abdallah, Amed, et les autres, aborde le difficile problème du statut des travailleurs immigrés et 
conduit le spectateur à s'interroger sur ce qu'on pourrait appeler "le syndrome de la transplantation : pourquoi certains travailleurs immigrés, après quelques années d'une vie tout à fait "normale" à l'étranger, perdent-ils en quelque sorte la raison à la suite d'un accident de travail?» (notice de présentation du film).

Mêlant alternativement, selon une progression dramatique à l'intérieur de chaque série, des séquences d'entretien des malades avec le médecin (qui nous donnent à voir leur malaise à travers leur gestuelle tout autant que leur discours ou le témoignage de leurs proches), des séquences d'interview de divers spécialistes (qui apportent des explications de type psychosociologique,

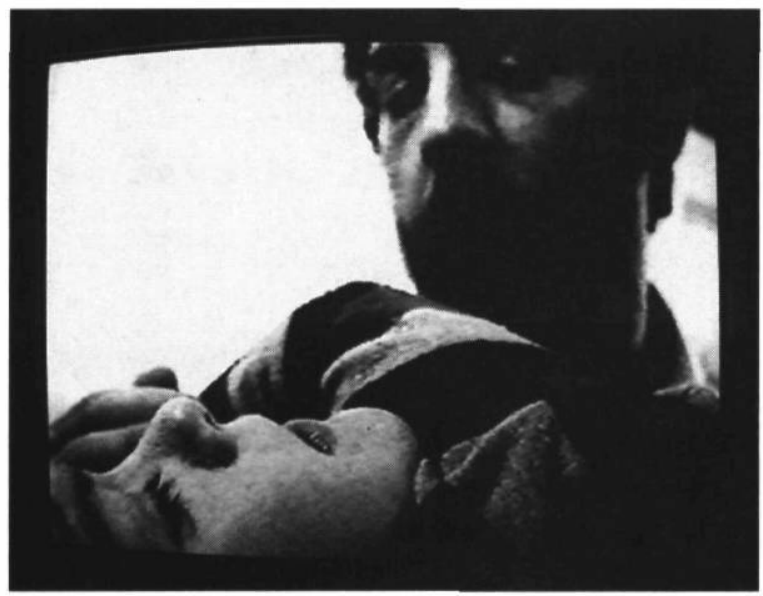

SAID ABDALLAH, AMED, ET LES AUTRES de Giovani Lentini (1982)

institutionnelle et sociopolitique) et des séquences "fictionnelles» (pour nous faire partager l'imaginaire de l'immigré, qui nourrit sa vie quotidienne), ce document multiplie les positionnements possibles du spectateur en jouant de la palette des dispositifs disponibles pour faire comprendre, modifier, déplacer les représentations du grand public vis-à-vis des immigrés.

Les cinq séquences, toujours spontanément identifiées par les spectateurs comme "fictionnelles», concernent cinq mises en 
scène d'un personnage joué par le même acteur professionnel, évoquant diverses situations où se joue, avec la voix off d'un immigré maghrébin s'appropriant les paroles d'un texte de Tahar Ben Jelloun, quelque chose comme la revendication d'un droit à la différence (qu'il s'agisse de sa façon de percevoir et de ressentir ou de la forme de son corps), ou comme le désir d'intégration pour avoir le droit de vivre comme tout le monde tout en sauvegardant "son paysage intérieur" — autrement dit son imaginaire, que la caméra ne pouvait rendre accessible ni à travers les longs plans séquences d'entretiens avec les travailleurs accidentés ni à travers les scènes d'interview. D'un point de vue narratologique, on peut dire qu'une diégèse s'instaure ainsi par les relations entre le personnage-immigré-type, le décor et les objets qui l'entourent, et «le documentaire met[tant] en jeu une image [et un son] qui renvoie[nt] censément au regard d'une instance interne au monde décrit, (...) glisse véritablement vers la fiction" (Jost, p. 48).

Ainsi placées dans le contexte de séquences d'entretiens et d'interviews, ces images non pas issues du monde réel mais, comme toute fiction, simulant un monde, à savoir ici la façon dont l'immigré vit, en imagination, sa différence - cette simulation subjective en quelque sorte - loin de nous éloigner du "témoignage du monde", en renforce la véracité, en accroissant notre relation émotionnelle au personnage réel, et nous donne à sentir sinon à comprendre sa souffrance. Les mini-récits et les procédés fictionnels sont utilisés ici à des fins rhétoriques, pour renforcer l'impact documentaire, pour un "plus d'effet de réel».

\section{DE LA TENSION FICTIONNALISANTE COMME MANIFESTATION DU «POINT DE VUE» ASSUMÉ}

Dans le deuxième documentaire, il n'y a ni acteur professionnel, ni décor, ni texte littéraire, ni voix off: seulement des images et des bruits d'ambiance, des bribes de conversations, des cris de douleur ou des souffles d'effort qui permettent tantôt d'anticiper, tantôt de revivre les situations difficiles que traverse le jeune Éric.

Éric ou L'oiseau bleu présente, en effet, un Institut médicopsycho-pédagogique (à Anheit, en Belgique), en restituant des moments de la vie du jeune handicapé mental profond, dans 
cette institution. Les presque 20 minutes du film nous permettent de voir "par les yeux d'Éric", un des petits pensionnaires, mais aussi de "percevoir" ce qu'il ressent, à travers ce que son corps subit et produit entre les mains savantes et encourageantes des thérapeutes, et à travers ses relations avec les autres enfants et les adultes — sa mère qui le mène à la camionnette de ramassage, le moniteur qui l'accueille au Centre, la secrétaire qui enregistre son dossier, etc. C'est comme si, au réveil, Éric revivait (flashback) ou anticipait (flash-forward) chaque moment de sa journée passée (ou à passer) dans l'institution, avec une mémoire à vif, faite de cris, de gestes, de souffrances mais aussi d'efforts récompensés, de triomphes.

Il est intéressant d'apprendre que ce film, primé au Festival de Tokyo en 1983 (prix d'excellence), a été refusé par l'institution commanditaire dans la mesure où il n'entrait pas dans le moule attendu et constituait (surtout à l'époque) un antimodèle : il ne contient ni discours de responsables, ni explications détaillées sur le parcours du jeune handicapé, ni témoignages moralisateurs sur le dévouement du personnel, le malheur de ces pauvres enfants, leur avenir, leurs parents... seulement un travail du matériau filmique hétérogène qui permet au spectateur de comprendre "de l'intérieur", comme on dit, ce que peut vivre l'enfant dans l'institution.

Là, en effet, c'est l'ensemble des événements, objets et personnages réels qui nous sont donnés à voir et à entendre, comme si c'était par les yeux, les oreilles et tout le corps d'Éric, comme si le réalisateur s'était mis à sa place et nous invitait à nous $y$ mettre. Le récit, si l'on veut bien en voir un ${ }^{13}$ avec un début, fait à partir de l'arrivée d'Éric à l'Institut et d'une succession d'activités évoquées, construit une histoire, celle de la journée d'Éric à L'Oiseau bleu, par le biais de la narration de faits et gestes réels, produite par "un acte narratif" assumé non par le locuteur second qu'est le commentateur comme c'est le cas le plus souvent dans un documentaire - puisqu'il n'y a pas de commentaire mais par le locuteur premier, narrateur implicite qui organise le film, coïncidant avec l'auteur. Les rapports entre histoire et récit s'explicitent nettement par rapport aux trois axes de l'ordre, de la durée et du mode (Genette) : les 20 minutes du monde reconstitué 
correspondent à une durée diégétique d'au moins 24 heures de la vie de l'enfant : le déroulement du récit ne reprend pas tous les épisodes de la journée et sans doute pas obligatoirement dans l'ordre dans lequel ils se sont déroulés, mais évoque plus une journée type qu'une journée dans son déroulement réel; quant au mode narratif ou "point de vue" qui guide la relation des événements, le phénomène de focalisation (le qui-voit?) et plus généralement d'énonciation (le qui-parle?), il est particulièrement intéressant à étudier ici.

En réalité, alors que tout le film donne l'impression que l'on vit à travers les diverses perceptions d'Éric, très peu de plans sont

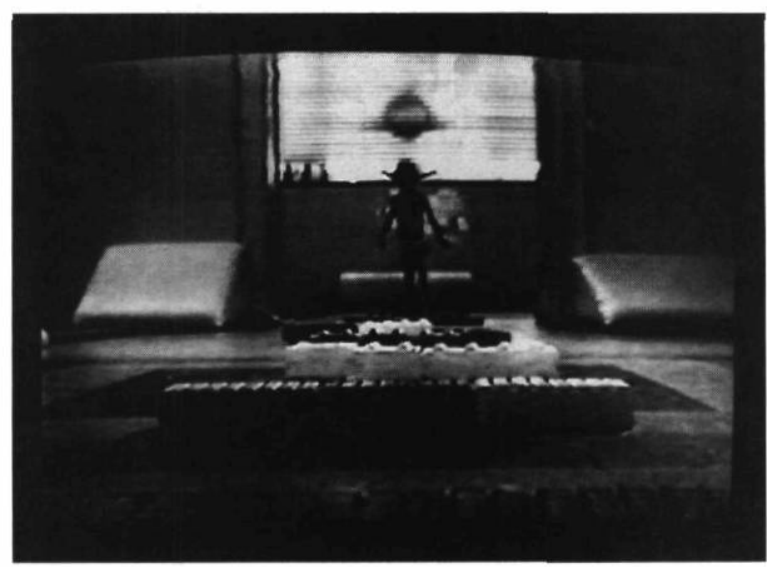

ERIC OU L'OISEAU BLEU

de Jean-Claude Riga (1983)

en ocularisation interne (secondaire ou primaire, c'est-à-dire référés directement ou par montage au personnage d'Éric) — un seul exemple, la séquence dans le grand magasin où nous voyons acheteurs, caisses et chariots avec les yeux d'Éric, dans un axe œil-caméra à la hauteur du petit handicapé. La plupart des autres plans sont en ocularisation zéro non marquée, c'est-à-dire qu'il n'y a pas d'observateur diégétique - la seule exception étant ce qu'en suivant François Jost, on pourrait appeler une 
"énonciation narrative» (une ocularisation zéro marquée), soit le passage, au milieu du film à peu près, d'un plan large et frontal sur la succession des cubes que le jeune handicapé a à franchir pour s'entraîner à maîtriser sa marche, à un gros plan, en légère plongée, sur les cubes et les pieds de l'enfant, qui nous donne l'impression de suivre l'effort fait par Éric, voire de le partager ou de l'aider à triompher des difficultés, impression renforcée par les encouragements de plus en plus pressants donnés par la voix off du thérapeute. Comment donc, avec cette majorité de plans en ocularisation zéro non marquée peut être donnée cette impression que ce que l'on voit, entend et ressent, on le perçoit par les yeux et les sens d'Éric lui-même?

De plusieurs façons, me semble-t-il. Au plan de l'image, par le retour, régulier (huit fois), comme un leitmotiv, de ce plan de réveil où Éric, couché dans son lit, vu de trois quarts, entrouvre progressivement les yeux, et ce d'autant plus que, progressivement, les gestes qu'il fait ou les cris qu'il profere dans chacun de ces inserts renvoient directement à ceux que l'on vient de (ou que l'on va) voir faire, par lui-même ou par un de ses semblables, lors d'une activité thérapeutique au Centre (raccord dans le mouvement, dans la couleur ou dans le cri) : image d'identification d'Éric, certes, mais qui devient bientôt symbole de l'imaginaire du jeune handicapé. Ensuite, au plan sonore, par le maintien, tout au long du film ou presque et quels que soient les autres bruits d'ambiance, du souffle (amplifié) de la respiration d'Éric, souffle long ou court de l'effort, de la vie ou tout simplement du rapport à l'univers. Enfin, par un asynchronisme presque systématique des éléments de la bande-image par rapport à la bande-son qui renforce cette impression de flash-back ou de flash-forward et surtout qui contribue à nous faire passer insensiblement, comme Éric qui se réveille, du monde du rêve ou de l'imaginaire à celui de la réalité : plus qu'un récit, "c'est le flux et le reflux de sons, de bruits, de gestes, de regards et d'émotions partagés par les enfants, le personnel éducatif, le réalisateur et les spectateurs potentiels" (Welkenhuysen, p. 36) ${ }^{14}$ qui structure tout le film.

D'ailleurs, le film se termine à peu près comme il a commencé, avec le trajet de la camionnette qui amène les enfants à L'Oiseau bleu, semblable à cet anneau de Mœbius qui n'a ni commencement 
ni fin, comme sans doute ce long et patient travail du thérapeute ou del'enfant, en vue d'un progrès toujours espéré et toujours à venir.

On peut dire que la fiction s'est introduite tout entière dans ce documentaire dans la mesure où celui-ci «tente de constituer un monde cohérent et consistant, c'est-à-dire où tout événement concourt à l'établissement d'un sens unitaire" (Jost, p. 45), à savoir l'incohérence, disons plutôt la cohérence différente de la nôtre, du monde du handicap. Mais si nous préférons parler de tension fictionnalisante plutôt que de fiction, c'est, d'une part, parce que chacune des séquences est prélevée sur le monde non pas inventé mais réellement vécu par Éric (le monde «donné à voir" par le documentaire continue à exister après le travail du documentariste), même s'il est retravaillé par la diégèse, et surtout parce que toute cette mise en scène ou en cadre ainsi que ce montage audiovisuel sont opérés au bénéfice non pas finalement d'une histoire racontée (celle de la journée dans une institution médico-psycho-pédagogique), mais au bénéfice du "témoignage du monde", d'un monde particulier qui est celui de l'enfance handicapée, le monde "supposé être celui de l'enfant handicapé» serait-il préférable de dire.

La plupart du temps, en effet, ce document n'est pas perçu comme "objectif" (on dit : "Qu'est-ce qui prouve que les images proposées par le réalisateur, qui sont souvent jugées "poétiques", correspondent réellement au monde imaginaire du handicapé mental?»). La subjectivité n'est jamais ici revendiquée. Elle est en revanche pleinement assumée par le réalisateur et proposée au spectateur, par "le grand imagier» ou "méganarrateur», selon une modalité d'énonciation marquée au plan visuel mais surtout audiovisuel, par le désynchronisme systématique et l'irruption musicale d'un saxophone : c'est le point de vue du cinéaste, opposé à celui de la caméra de surveillance que l'on voit très clairement dans la séance du magasin, «refus de l'écriture documentaire dominante, prétendument objective et neutre, se distanciant de la réalité pour mieux la reproduire sous forme d'une narration linéaire, construite de manière analytique et impersonnelle" (Welkenhuysen, p. 34).

Ainsi donc, bien que "masqué» au plan de l'énonciation (Jost), pour s'avancer, le réalisateur n'en a-t-il pas moins pris des risques. 
Celui, institutionnel, de voir refusé son film qui ne cadrait plus avec le modèle attendu pour ce genre de documentaire à propos d'une institution; celui, esthétique, d'un documentaire témoignant d'un point de vue d'auteur; celui, éthique, d'un engagement par rapport à ce monde du handicap dont le réalisateur voudrait donner une autre représentation que celle qui a cours habituellement, et donc par rapport à une certaine conception du «cinéma du réel».

\section{DE la FiCTIONNALISATION COMME SUBJECTIVITÉ REVENDIQUÉE ET OBJECTIVÉE}

Mais la fiction et le point de vue subjectif qui l'accompagne ne sont pas toujours le fait d'une simulation subjective ni seulement la manifestation d'un point de vue assumé. Ils peuvent tisser l'ensemble du propos filmique comme une voix subjective, revendiquée comme telle, et qui nous invite non pas à une fusion mais à une confrontation entre le "sujet" et l'«objet", entre le monde réel et la façon dont le vit celui qui en témoigne. C'est peut être une des façons de ne pas tomber dans le piège du "documentaire de création" où le réalisateur s'abandonne à sa subjectivité sans s'interroger sur les relations qu'il entretient avec ce monde dont il veut témoigner.

C'est le cas de ce troisième documentaire qu'avec Bill Nichols on pourrait ranger - si l'on tient aux classements - dans la catégorie des documentaires "réflexifs»" : avec une masse d'images et de sons y compris d'archives tournées au jour le jour, pendant six semaines de grève générale dans la sidérurgie à Liège, il met en question non seulement le système de représentation syndicale mais plus pertinemment, pour le propos qui nous intéresse, la mise en forme documentaire.

La réalisatrice, en effet, «agence des images fournies par la réalité matérielle (le monde de la sidérurgie en grève) qui sont entièrement transformées par un nouvel engagement dans un monde imaginaire" (Welkenhuysen, p. 34) qui est le sien, sans gommer, dans l'énoncé lui-même, comme cela se passe souvent dans les reportages ou les documentaires de création, les traces du locuteur. Paysage imaginaire - comme le titre l'indique met en évidence la part de l'imaginaire dans notre perception du réel (on pense au Chris Marker de Lettres de Sibérie ${ }^{16}$ ) et, à 
travers des indices textuels, affirme que ce qui est donné à voir et à comprendre, dans un documentaire aussi, c'est le regard de quelqu'un sur les choses.

Donnons-en quelques exemples. La première séquence du film consiste en une succession de plans larges de nuit, de port, de feu alternant avec des gros plans de jour sur des colonnes de fumée, des architectures industrielles, des ciels rougeoyants, pendant qu'une puis deux voix off hésitantes d'enfant déchiffrent un extrait des 500 millions de la Begum (Jules Verne, 1879), où il est question d'une description de la ville de l'acier (Stahlstadt) : on ne sait de quoi il va être question sinon d'un monde inconnu ou mal connu, qui reste à découvrir. Apparaît alors dans la séquence suivante, à travers un rétroviseur, les murs d'une usine longuement parcourus, pendant que se superpose aux voix d'enfants celle, féminine, qui égrène les noms de la géographie industrielle de la Wallonie et tandis qu'on peut lire, en alternance, apparaissant et disparaissant lettre par lettre, les mots "paysage imaginaire», "usine», «intérieur", "extérieur", "magie rouge", puis "colère d'acier", juste au moment où on commence à entendre un syndicaliste qui sera bientôt en voix on : le passage de l'inconnu au connu, de l'extérieur vers l'intérieur se fait progressivement, avec des choix (de réalisation) que rappelle le cadre du rétroviseur (un des procédés d'énonciation repéré par Christian Metz dans L'Énonciation impersonnelle ou le site du film, et qui sera repris plusieurs fois dans la suite du document, jeux de glaces de cabines téléphoniques et de rétroviseurs dans le car qui emmène les ouvriers vers la manifestation). Viendront ensuite les images d'archives de divers moments et lieux de la manifestation ouvrière (sans oublier la bière et les frites, Belgique oblige!) avec discours ou interviews de grévistes et commentaires de la radio ou de la télévision (toujours ce thème de la représentation) alternant avec de superbes plans de coulée fumante et d'ouvriers sidérurgistes au travail, tandis que s'amorce une tentative d' "explication" de l' "exaspération" conduisant à l' "explosion" (mots apparaissant à l'écran) comme la "grève" se transfomant momentanément en "trève" pour que se poursuivre le "rêve" de la "coulée continue» réclamée par les grévistes et symbole du sauvetage de la sidérurgie wallonne. 
Dans la dernière séquence, sur fond de reprise de la musique de Stockhausen (Stimmung), on aperçoit un téléviseur - écran dans l'écran - (et l'ombre d'un cameraman) qui déverse les paroles du syndicaliste du début, demandant qu'on continue à lui accorder la confiance, alors qu'on parcourt l'usine qui semble bien abandonnée et que reprend progressivement la lecture enfantine du texte de Jules Verne rappelant la difficulté pour les reporters de briser l'interdiction d'entrer dans l'usine...

Autant d'indices textuels de mise en question de la représentation, notamment audiovisuelle, de revendication de la place du témoin dans la restitution de l'événement, d'explicitation de la

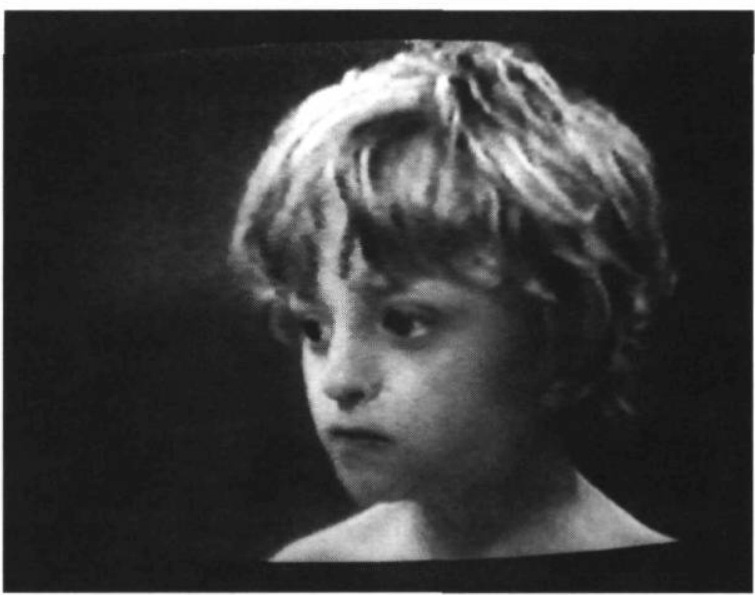

PAYSAGE IMAGINAIRE

de Nicole Widart (1992)

position idéologique de l'énonciateur qui sait qu'il ne peut parler "à la place» des ouvriers grévistes, sinon par un tour de passepasse, comme on dit - grâce à "la formule magique», disait Jules Verne dans le texte lu par la voix enfantine du début ou au moins «une autorisation dûment timbrée, signée et paraphée».

Ainsi, ni documentaire subjectif — où "c'est la subjectivité du réalisateur ou des personnages filmés qui l'emporte en dernière analyse" —, ni fiction documentée — où "c'est l'opération de 
transformation fictionnelle sur les éléments documentaires" qui l'emporte (Leblanc) - , ces trois documentaires tentent au contraire d'approfondir le rapport film/réalité: le premier en exprimant, à travers des mini-récits (mise en scène, acteur, décor, dialogue ou voix intérieure), la dimension subjective de l'imaginaire si souvent oubliée dans l'analyse des raisons (objectives) du syndrome de transplantation des travailleurs immigrés accidentés; le second en assumant, par une tension fictionnelle où le référentiel est repris par l'imaginaire et reconstruit la réalité, à travers le point de vue du réalisateur dont "chaque plan, avant d'être incorporé dans une séquence, se voit déjà stratifié au tournage par la collision marquée d'un réel et d'un regard" $(\text { Fargier })^{17}$; le troisième, en revendiquant en quelque sorte, par une mise en scène de la place de soi dans la perception de l'événement, la dimension subjective de toute représentation. Tous trois échappent, de ce fait, "au mythe de la transparence» qui a toujours été la grande tentation du cinéma, documentaire en particulier. Les informations para et péritextuelles que nous avons sur eux, ainsi que la connaissance directe que nous en avons contribuent, sans aucun doute, à faciliter cette perception : ils appartenaient à un groupe de réalisateurs à la recherche d' "une autre télévision" et pour qui, comme l'avait dit Godard, «il ne s'agit pas de faire des films politiques mais de faire politiquement des films».

Ces divers exemples montrent bien comment la subjectivité et les phénomènes d'identification et de fictionnalisation qu'ils suscitent, loin de s'opposer à la fonction "témoignage du monde» du documentaire, la renforce en lui apportant "un plus de réel": ils apportent, comme l'a dit Bill Nichols, "une forme spécifique et revitalisante de référentialité» ${ }^{18}$. Il y a là une tendance qui s'est récemment accentuée et que d'aucuns relient directement à l'évolution de la pensée postmoderne. Le fait pourtant n'est pas nouveau, et il suffirait d'évoquer, dans l'histoire du cinéma, ces mouvements-repères accompagnés d'expressions clés ${ }^{19}$ qui montrent comment ces deux modalités de relation au réel, documentaire et fiction, se sont souvent combinées, selon des degrés d'interpénétration divers et variables avec les époques, les lieux et les secteurs d'intervention du documentaire. 
Mais ce qui est nouveau, c'est le plus grand degré d'acceptabilité de cette dimension subjective dans un genre qui s'est longtemps et généralement identifié à la recherche de l'objectivité. Acceptation toute relative encore, si l'on songe à la polémique suscitée au États-Unis par le film de Michael Moore, Roger and Me (il est le moi du film), sur les conséquences catastrophiques, pour sa ville natale Flint (Michigan), de la décision du PDG, Roger Smith, de transférer la production de la compagnie au Mexique, où la main-d'œuvre est moins chère et moins syndiquée - polémique qui mettait en cause le comité de sélection des films pour les Oscars qui, de l'aveu même du président de l'International Documentary Association, est depuis longtemps "plus enclin à privilégier le sujet que l'imagination cinématographique, ce qui tend à renforcer l'image d'une discipline méritoire mais peu excitante" (Le Monde, 22 mars 1990).

Si subjectivité et identification sont beaucoup plus rarement exploitées dans le documentaire que dans la fiction, comme le remarque très justement Bill Nichols, il serait temps de prendre conscience que cette séparation n'est pas liée aux caractéristiques propres du genre, mais est, sans doute, le fait de conventions esthétiques et donc de circonstances historiques.

\section{Qu'est-ce qu'un documentaire?}

Qu'est-ce donc ce "genre" qui, à la fois, s'affiche empiriquement, historiquement et institutionnellement si clairement, qui ne semble exister que négativement par opposition à la fiction et qui résiste si fort à toute tentative de formalisation théorique?

Un genre, comme l'a pertinemment montré Jean-Louis Leutrat à propos du western, est "ce que, collectivement, on croit qu'il est, à un moment donné» (p. 27). La conception du documentaire a en effet varié avec les lieux et les époques, et le documentaire n'occupe pas un territoire fixe, ni comme pratique ni comme concept, ni comme théorie.

À l'évidence, le documentaire existe comme "genre», dans l'institution (cinématographique, télévisuelle et même vidéographique, voire infographique) ainsi que dans l'expérience commune, mais à l'évidence aussi, il est difficile de le maintenir au plan théorique, comme genre opposé à la fiction, sans autre 
forme de procès, ne serait-ce que parce qu'il n'y a pas de coïncidence possible entre image et réalité (tout film est de fiction) et parce que l'imaginaire participe de notre perception du réel, avec ou sans caméra. Il n'y a pas d'un côté le cinéma (ou l'audiovisuel) comme moyen de représentation du réel et, de l'autre, le cinéma comme moyen d'expression au service de l'imaginaire, mais un langage et une technique qui ont leurs spécificités, l'un et l'autre pouvant être mis au service soit d'un discours sur la réalité, soit d'une histoire racontée.

Et s'il est vrai que le signifiant du cinéma «se prête mieux à la fiction" (sous-entendu que celui du thêâtre, par exemple, où la représentation est "réelle») "dans la mesure où il est lui-même fictif et absent" et que le réel au cinéma n'est jamais qu'un "effet de réel", cela ne suffit pas pour en conclure que le documentaire est une fiction comme les autres.

Il n'est donc pas possible de nier les différences entre fiction et documentaire pour des raisons aussi bien pragmatiques que théoriques. Plutôt que de proposer une définition ontologique du documentaire, mieux vaut tenter d'en approcher les spécificités de trois points de vue : du côté du réalisateur, car un auteur de roman ou de film ne se construit pas à partir de sa seule production mais à partir de tout le processus qui y conduit-sa maîtrise de l'outil, qui permet une construction et non une traduction de la réalité, et son engagement à la fois personnel et idéologique par rapport à cette réalité dont il veut rendre compte; du côté du texte, car si le documentaire peut emprunter ses marques à la rhétorique et aux principes fictionnels et s'il peut être narratif, son économie générale ressortit à une autre pertinence plus argumentative que narrative; du côté du spectateur enfin, car si «l'effet fiction" au cinéma instaure un régime particulier de "croyance», comme cela a été bien montré, reste à analyser ce régime particulier de persuasion, mieux de "conviction" qu'instaure l'«effet de réel» du documentaire et qui n'est pas, comme on l'a dit trop souvent - là encore par opposition à la fiction - exempt de "plaisir", ce plaisir du connaître ou epistephilia. 
1 Définition du Vocabulaire de l'éducation (Paris : PUF, 1979) ou dans Bessey et Chardon, Dictionnaire du cinéma et de la télévision (Paris : Pauvert, 1965) : "Genre cinématographique rejetant la fiction pour rendre présente la seule réalité."

2 Depuis la disparition de la "première partie" de programme de la séance de cinéma, à la fin des années 50 , la diffusion à la télévision a constitué la source principale de diffusion du documentaire. Depuis 1991, une nouvelle association, "Documentaire sur grand écran" organise, à Paris (à L'Entrepôt et au cinéma Utopia) et en province, des programmations de films documentaires.

3 Quelques exemples parmi d'autres : à propos d'Allemagne, année zéro de Godard : "Dans ce film qui n'est ni une fiction ni un documentaire mais une déambulation, il fait entrer un personnage de fiction mythique" (Jacques Siclier dans Le Monde, 12 août 1993); à propos des Chroniques de l'hôpital d'Armentières de Daniel Karlin et sous le titre "Le feuilleton-documentaire ou l'éloge de l'ordinaire", Laurence Folléa précise que "sur un fil qui balance entre réalité et fiction (...) le feuilleton-fiction, romance de la vie, est devenu feuilleton-réalité, montage du quotidien, une sorte de reality-show à l'envers" (Le Monde, 22 septembre 1993); le même journal (supplément RTV du 27 décembre 1993 au 2 janvier 1994) annonce que pour reconstituer l'insurrection vendéenne, "Jacques Dupont s'est appuyé sur des documents historiques; entre fiction et documentaire, son film crée un effet de réel saisissant" (c'est nous qui soulignons).

4 Saîd, Abdallah, Amed et les autres, réalisateur Giovani Lentini, 1982, 20' couleur; Éric ou l'oiseau bleu, réalisateur Jean-Claude Riga, 1983, 20' couleur; Paysage imaginaire, réalisatrice Nicole Widart, 1982, 30' couleur, tous trois produits et diffusés par la chaîne liégeoise câblée Canal Emploi, qui n'existe plus à présent mais qui a représenté, dans les années 80 , un haut lieu de réflexion sur le traitement documentaire ou "cinéma du réel", avec laquelle j'ai eu le plaisir de travailler et à laquelle j'aime rendre hommage.

5 On donne souvent l'exemple du western, mais Jean-Louis Leutrat l'a montré magistralement à propos des films de l'Ouest des années 20 in Cinémas et réalités (Saint-Étienne : université de Saint-Étienne, 1984) pp. 161-172.

6 On se souvient que pour Vertov, le traitement documentaire, celui des faits, n'est pas un pis-aller mais une priorité, contre la "ciné-sorcellerie" et la "ciné-mystification" pour "ouvrir les yeux des masses populaires sur le lien qui unit les phénomènes sociaux et visuels mis en lumière par les caméras"; quant à Grierson, il prône un cinéma "de service public", "antistudio", "anti-star-system", tourné en "décor naturel" qu'il considère "comme une chaire et (dont) il se sert en propagandiste".

7 On peut évoquer la tradition des "actualités reconstituées" inaugurée par Méliès, dans son studio embryonnaire de Montreuil, qu'il s'agisse de la guerre gréco-turque, de L'Explosion du cuirassé Maine en rade de la Havane, réalisé au moyen de prises de vues devant un aquarium, ou de la célèbre reconstitution "antéhistorique", comme on l'a dit du Couronnement d'Édouard VII, tourné en 1902, avant la cérémonie elle-même.

8 "Tout message par le truchement duquel une histoire, quelle qu'elle soit, est communiquée devrait à bon droit être considéré comme un récit.» André Gaudreault, Du littéraire au filmique. Système du récit (Paris/Québec : Méridiens Klincksieck/ Presses de l'université Laval, 1988) p. 84.

9 Je n'entre pas ici dans le débat entre Jost et Metz sur le fait de savoir s'il faut distinguer la subjectivité comme phénomène langagier et l'étude des instances narratives ou s'il faut dire que la narration n'est qu'un mot spécialisé pour désigner l'énonciation narrative. Voir à ce sujet François Jost, Un monde à notre image. Énonciation, Cinéma, Narration (Paris : Méridiens Klincksieck, 1992), chapitre I. 
10 Dans son troisième chapitre, William Guynn montre que dans les documentaires (classiques), la proportion de syntagmes narratifs/non narratifs est inversée par rapport à la fiction, mais que la fonction discursive n'est pas seulement assurée par le commentaire, comme il le prouve par l'exemple du film de propagande sans commentaire, Listen to Britain (Humphrey Jennings, 1942) où cette fonction est assurée par les emblèmes visuels (le canon = courage militaire, et le violon = la vie domestique, le sentiment, le bonheur) et les marques d'énonciation. Voir William Guynn, A Cinema of Nonfiction (London/Toronto : Associated University Press, 1990).

11 Les "faux" premiers mètres des maîtres du documentaire (Joris Ivens, Dziga Vertov, Jean Rouch, Frédérick Wiseman, Nagisa Oshima), à partir d'une commande de la bibliothèque de Beaubourg, qui furent les seuls à se rendre compte du canular... mais vinrent féliciter le hardi réalisateur qui commente ainsi cette expérience : "(...) le problème du documentaire, c'est qu'il est soi-disant plus "vrai" que la fiction, mais, pour reconstituer le réel, sur support pellicule, il faut construire un dispositif : dans le cinéma documentaire, il y a aussi manipulation d'images et de sons" (Dominique Biton, "Un pastiche : "Premiers mètres", de Pierre-Oscal Lévy : "Dans le documentaire aussi, il y a manipulation d'images et de sons" ", Le Documentaire français (sous la direction de René Prédal), CinémAction no 41 (1987) p. 112.

12 C'est une question qui fait, en effet, l'objet d'un livre sur le documentaire à paraître chez Colin, dans la collection "Cinéma et audiovisuel" dirigée par R. Odin.

13 Sur la question très controversée "Qu'est-ce qu'il faut demander exactement à un texte pour qu'on se permette de lui accorder un statut narratif?", voir André Gaudreault, ibid., pp. 63 et suivantes.

14 Commentaire de Guido Welkenhuysen dans un mémoire (année 83-84) en vue d'une licence en communication sociale de l'École de cinéma flamande (RITCS), auquel nous empruntons diverses citations, où il se livre, à propos de ces trois documentaires, "à une approche de l'écriture télévisuelle à partir de la pratique de trois réalisateurs" qu'il a longuement interviewés.

15 Bill Nichols distingue en effet quatre catégories : le documentaire d'exposition (expository), d'observation (observational), d'interaction (interactive) et réflexif (reflexive), qui correspondent approximativement à une chronologie, même si les différents modes ont toujours cœxisté. Voir Bill Nichols, Representing Reality, Issues and Concepts (Indiana University Press : 1990) pp. 32-75.

16 La voix off du commentaire précise : «L'objectivité non plus n'est pas juste. Elle ne déforme pas la réalité sibérienne mais elle l'arrête le temps d'un jugement et par là, elle la déforme quand même. Ce qui compte, c'est l'élan et la diversité. Ce n'est pas une promenade dans les rues d'Irkoutsk qui vous fera comprendre la Sibérie. Il y faudrait un film d'actualités imaginaires, prises aux quatre coins du pays.”

17 "Sans images préconçues", article de Jean-Paul Fargier à propos de Johan Van der Keuken, Cahiers du cinéma no 289.

18 Bill Nichols dit que "the subjective moment (...) offer a distinct and revitalizing form of referentiality to today's documentary" (p.160), des trois autres modalités de la référentialité étant l'"excès", le "lien indexical" et "la reconnaissance des faits du monde réel". Voir à ce sujet Bill Nichols, ibid., chapitre V: "Sticking to reality, rhetoric and what exceeds it", pp. 141-165.

19 De "la mise en scène documentaire" chère à Flaherty au "Je ne fais pas de l'art passif" du Hollandais Joris Ivens, en passant par "le traitement créatif de l'actualité» de Grierson ou le célèbre "point de vue documenté» de Jean Vigo, voir G. Jacquinot, "Le Genre documentaire existe-t-il?", Panorama des genres au cinéma (sous la direction de Michel Serceau), CinémAction no 68 (1993) pp. 162-172. 


\section{OUVRAGES CITÉS}

Daney, Serge. Ciné-Journal. Paris : Cahiers du Cinéma, 1986.

Fargier, Jean-Paul. "Sans images préconçues", Cahiers du cinéma n 289.

Jost, François. "Mon tout est une fiction». La Licorne no 17 (1990) pp. 39-50.

Leblanc, Gérard. "La Réalité en question". Le Documentaire français (sous la direction de René Prédal), CinémAction no 41 (1987) pp. 36-45.

Leutrat, Jean-Louis. "1895 Films», Iris vol. 1 no 1 (1983) pp. 19-38.

Metz, Christian. Le Signifiant imaginaire. Psychanalyse et cinéma. Paris : Christian Bourgois, 1977.

Welkenhuysen, Guido. Le Cran du petit écran : approche de l'écriture télévisuelle à partir de la pratique de trois réalisateurs de Canal Emploi. Bruxelles: RITCS, 2 vol., 1983-1984. 